\title{
Understanding the origins of the heliosphere: integrating observations and measurements from Parker Solar Probe, Solar Orbiter, and other space- and ground-based observatories
}

\author{
M. Velli ${ }^{1,7}$, L. K. Harra ${ }^{2,3}$, A. Vourlidas ${ }^{4}$, N. Schwadron ${ }^{5}$, O. Panasenco ${ }^{6}$, P. C. Liewer ${ }^{7}$, D. Müller ${ }^{8}$, I. Zouganelis ${ }^{9}$, \\ O. C. St Cyr ${ }^{10}$, H. Gilbert ${ }^{10}$, T. Nieves-Chinchilla ${ }^{10}$, F. Auchère ${ }^{11}$, D. Berghmans ${ }^{12}$, A. Fludra ${ }^{13}$, T. S. Horbury ${ }^{14}$, \\ R. A. Howard ${ }^{15}$, S. Krucker ${ }^{16}$, M. Maksimovic ${ }^{17}$, C. J. Owen ${ }^{18}$, J. Rodríguez-Pacheco ${ }^{19}$, M. Romoli ${ }^{20}$, \\ S. K. Solanki ${ }^{21,27}$, R. F. Wimmer-Schweingruber ${ }^{22}$, S. Bale ${ }^{23}$, J. Kasper ${ }^{24}$, D. J. McComas ${ }^{25}$, N. Raouafi ${ }^{4}$, \\ V. Martinez-Pillet ${ }^{26}$, A. P. Walsh ${ }^{9}$, A. De Groof ${ }^{9}$, and D. Williams ${ }^{9}$
}

(Affiliations can be found after the references)

Received 24 April 2020 / Accepted 9 June 2020

\begin{abstract}
Context. The launch of Parker Solar Probe (PSP) in 2018, followed by Solar Orbiter (SO) in February 2020, has opened a new window in the exploration of solar magnetic activity and the origin of the heliosphere. These missions, together with other space observatories dedicated to solar observations, such as the Solar Dynamics Observatory, Hinode, IRIS, STEREO, and SOHO, with complementary in situ observations from WIND and ACE, and ground based multi-wavelength observations including the DKIST observatory that has just seen first light, promise to revolutionize our understanding of the solar atmosphere and of solar activity, from the generation and emergence of the Sun's magnetic field to the creation of the solar wind and the acceleration of solar energetic particles.

Aims. Here we describe the scientific objectives of the PSP and SO missions, and highlight the potential for discovery arising from synergistic observations. Here we put particular emphasis on how the combined remote sensing and in situ observations of SO, that bracket the outer coronal and inner heliospheric observations by PSP, may provide a reconstruction of the solar wind and magnetic field expansion from the Sun out to beyond the orbit of Mercury in the first phases of the mission. In the later, out-of-ecliptic portions of the SO mission, the solar surface magnetic field measurements from SO and the multi-point white-light observations from both PSP and SO will shed light on the dynamic, intermittent solar wind escaping from helmet streamers, pseudo-streamers, and the confined coronal plasma, and on solar energetic particle transport.

Methods. Joint measurements during PSP-SO alignments, and magnetic connections along the same flux tube complemented by alignments with Earth, dual PSP-Earth, and SO-Earth, as well as with STEREO-A, SOHO, and BepiColumbo will allow a better understanding of the in situ evolution of solar-wind plasma flows and the full three-dimensional distribution of the solar wind from a purely observational point of view. Spectroscopic observations of the corona, and optical and radio observations, combined with direct in situ observations of the accelerating solar wind will provide a new foundation for understanding the fundamental physical processes leading to the energy transformations from solar photospheric flows and magnetic fields into the hot coronal plasma and magnetic fields and finally into the bulk kinetic energy of the solar wind and solar energetic particles.

Results. We discuss the initial PSP observations, which already provide a compelling rationale for new measurement campaigns by SO, along with ground- and space-based assets within the synergistic context described above.
\end{abstract}

Key words. solar-terrestrial relations - Sun: heliosphere - Sun: corona - solar wind - Sun: magnetic fields - Sun: atmosphere

\section{Introduction}

The solar magnetic field plays a defining role in the formation and structuring of the solar wind and the heliosphere. In the solar corona, closed magnetic field lines confine the hot plasma in the form of loops, while open magnetic field lines guide the expansion of the solar wind into interplanetary space. Which field lines open into interplanetary space and which field lines confine, at least partially, the coronal plasma is determined by the magnetic field dynamics. This is driven by the large-scale solar dynamo resulting in the emergence of sunspot and active regions; large-scale solar surface flows (solar differential rotation and meridional circulation); and the smaller scale turbulent convective motions below and at the photosphere. It is here that emerging magnetic field and the continuous buffeting of field line footpoints by photospheric motions converts, transports, and stores the energy that leads to coronal heating, the acceleration of the solar wind, and the formation of the heliosphere itself.
Two recently launched space missions, Parker Solar Probe (PSP; Fox et al. 2016) and Solar Orbiter (SO; Müller et al. 2020), are devoted to understanding the plasma physics at the heart of the Sun's magnetic activity. At the same time, a number of Earth-orbiting assets, from the Solar Dynamics Observatory (Pesnell et al. 2012) to the IRIS spectroscopic mission (De Pontieu et al. 2014) and the Japanese Hinode satellite (Kosugi et al. 2007), provide quasi-continuous observations of the solar atmosphere. WIND and ACE (Stone et al. 1998) in orbit around L1 provide continuous in situ data. The STEREO-A (Solar TERestrial RElations Observatory - Ahead) spacecraft (Kaiser et al. 2008), orbiting the Sun from a vantage point presently trailing the Earth by about 75 degrees, provides both remote sensing and in situ data. In addition, the Daniel $K$. Inouye solar telescope (Tritschler et al. 2016), which saw first light in 2019, promises to provide new, fundamental, high-resolution measurements of the lower solar atmosphere and inner corona, providing both context and additional data points for PSP and SO observations. The 
current paper is devoted to a discussion of how joint observations by PSP, SO, and other ground- and space-based assets may revolutionize our understanding of solar activity, the corona, solar wind, the generation, acceleration, and transport of solar energetic particles (SEPs), and the fundamental physics of natural magnetized plasmas. This heliospheric exploration continues in the tradition of a field that provided the first collective multimessenger (Hudson \& Svalgaard 2019; McComas et al. 2017) studies of a physical system.

The PSP and SO top-level scientific objectives underline the main open scientific questions concerning the origins of the heliosphere:

PSP scientific objectives:

1. Trace the flow of energy that heats the corona and accelerates the solar wind.

2. Determine the structure and dynamics of the magnetic fields at the sources of the fast and slow solar wind.

3. Determine what mechanisms accelerate and transport energetic particles.

SO scientific questions:

1. How and where do the solar wind plasma and magnetic field originate in the corona?

2. How do solar transients drive heliospheric variability?

3. How do solar eruptions produce energetic particle radiation that fills the heliosphere?

4. How does the solar dynamo work and drive connections between the Sun and the heliosphere?

Though with slight differences in emphasis, PSP objectives (1) and (2) overlap with SO question (1), while SO objectives (2) and (3) are congruent with PSP questions (2) and (3). However, SO question (4) is unique to that mission and more specifically to its extended mission out of ecliptic phase, when observations will allow a view of the polar flows and magnetic field well beyond previous capabilities.

In the following sections, we discuss orbital characteristics and, briefly, the instrumentation on the PSP and SO missions, illustrating the joint observation opportunities provided by simultaneous in situ and remote-sensing instruments. We then provide specific examples of scientific advances provided by such joint observations before discussing the role played by other space- and ground-based assets. Subsequently, we present initial PSP observations and discuss how joint observations can be used to distinguish between different interpretations. Finally, we discuss the potential outcomes of heliospheric exploration over the next decade.

\section{Mission profiles and instrumentation of PSP and so}

Parker Solar Probe launched in the early morning of August 12, 2018, on a Delta 4 Heavy from Cape Canaveral, Florida, in the United States, carrying four suites of instruments: the FIELDS, WISPR, SWEAP, and IS $\odot$ IS investigations.

- The FIELDS experiment (Bale et al. 2016) measures magnetic and electric fields and their fluctuations in situ, and indirectly determines the electron density via plasma quasi-thermal noise spectroscopy. The investigation comprises two fluxgate magnetometers (MAG), a search coil magnetometer (SCM), and five electric antennas.

- The Integrated Science Investigation of the Sun energetic particle instrument suite (IS $\odot$ IS; McComas et al. 2016) measures the flux of energetic protons, electrons, heavy ions, and their distribution functions (tens of $\mathrm{keV}$ to $100 \mathrm{MeV}$ ). The instrument suite comprises two independent instruments (EPI-Hi and EPILo) covering different and overlapping energy ranges.

- The Solar Wind Electrons Alphas and Protons Investigation (SWEAP; Kasper et al. 2016) has two electrostatic analyzers (SPAN-A and -B) and a Faraday cup (SPC). This investigation measures the most abundant particles in the solar wind - electrons, protons, and helium ions - and determines their bulk properties such as velocity, density, and temperature as well as full 3D distribution functions.

- The Wide-field Imager for Solar Probe (WISPR; Vourlidas et al. 2016) white-light telescopes capture images of the solar corona and inner heliosphere. The experiment also provides images of the solar wind, shocks, and other structures as they approach and pass the spacecraft. This investigation complements the other instruments on the spacecraft by imaging the plasma that the other instruments will sample, as its field of view (FOV) is in the direction of spacecraft motion.

PSP carries out an extensive investigation of the inner heliosphere: to reach regions of space below $10 R_{S}$ from Sun center, PSP was launched into a Venus intercept trajectory that allowed a gravity assist about 6 weeks after launch for insertion into an elliptical orbit around the Sun with perihelion near $35.65 R_{S}$ (0.16 AU) and aphelion near $1 \mathrm{AU}$. After three perihelia (E1-E3) at $35.65 R_{S}$, a second Venus gravity assist occurred in December, 2019, before a fourth solar encounter (E4) on January 29, 2020 , at $27.89 R_{S}$. After encounter 5 (E5) on June 7, 2020, with the same perihelion, five subsequent Venus gravity assists will decrease the perihelion in stages to a final perihelion at $9.86 R_{s}$ $(0.0459 \mathrm{AU})$ in the final three orbits. The nominal mission lasts 7 years but carries sufficient fuel for continuation into an extended phase with the same $9.86 R_{s}$ perihelion every 88 days. Encounter periods are defined by the spacecraft being closer to the Sun than $0.25 \mathrm{AU}\left(53.76 R_{s}\right)$.

Throughout the seven-year nominal mission duration, the spacecraft will spend a total of 937 hours inside $20 R_{s}, 440$ hours inside $15 R_{s}$, and 14 hours inside $10 R_{s}$, sampling the solar wind in all its modalities (slow, fast, and transient) as it evolves with rising solar activity toward an increasingly complex structure (see Fig. 1). The final PSP orbit is inclined by only 3.4 degrees from the ecliptic plane and so will not sample the fast wind directly above the Sun's polar regions. However, the lack of in situ measurements of the fast wind above the polar regions is compensated by the relatively long time spent by PSP inside $20 R_{s}$. This allows prolonged measurement of the equatorial extensions of high-latitude and equatorial coronal holes. At a helioradius of $\simeq 35 R_{s}$, there are two periods per orbit (one inbound and one outbound) when PSP will be in quasi-corotation with the Sun and will cross a given longitudinal sector slowly. In these intervals, known as fast radial scans, the spacecraft will sample the solar wind over large radial distances within a given flux tube before moving across the sector. These measurements will yield additional information on the spatial/temporal dependence of structures in the solar wind and on how they merge in the inner heliosphere. SO, launched on February 10, 2020, on an ATLAS V also from Cape Canaveral, carries a comprehensive suite of both in situ and remote-sensing instrumentation, summarized below:

- The Energetic Particle Detector (EPD; Rodríguez-Pacheco et al. 2020) experiment will measure the composition, timing, and distribution functions of supra-thermal and energetic particles. The EPD consists of four separate sensors sharing a common data-processing unit and low-voltage power supply (CDPU/LVPS).

- The Extreme Ultraviolet Imager (EUI; Rochus et al. 2020) will provide image sequences of the solar atmospheric layers 
above the photosphere, thereby providing an indispensable link between the solar surface and outer corona that ultimately shapes the characteristics of the interplanetary medium.

- The magnetometer (MAG; Horbury et al. 2020) experiment will provide in situ measurements of the heliospheric magnetic field with high precision. In addition to studies of the near-Sun heliospheric plasma and fields, MAG will address how the magnetic field links into space and evolves over the solar cycle; how waves and turbulence are generated and dissipated; and how heliospheric structures develop in the inner Solars System.

- Metis, the Solar Orbiter visible light and ultraviolet coronal imager (Antonucci et al. 2020), will employ broad-band, polarized imaging of the visible K-corona and narrow-band imaging of the UV (HI Ly $\alpha, 121.6 \mathrm{~nm}$ ) corona that will be used to study the structure and dynamics of the full corona with unprecedented temporal coverage and spatial resolution.

- The Polarimetric and Helioseismic Imager (SO/PHI; Solanki et al. 2020) will provide high-resolution and full-disk measurements of the photospheric vector magnetic field and line-of-sight (LOS) velocity, as well as the continuum intensity in the visible wavelength range at a cadence of up to one set of observables per minute. The LOS velocity maps will have the accuracy and stability to allow detailed helioseismic investigations of the solar interior, in particular of the solar convection zone.

- The Radio and Plasma Waves (RPW: Maksimovic et al. 2020) experiment is unique amongst the SO instruments in that it takes both in situ and remote-sensing measurements. The RPW will measure magnetic and electric fields at high time resolution using a number of sensors and antennas, and will determine the characteristics of electromagnetic and electrostatic waves in the solar wind from almost direct current (DC) to $20 \mathrm{MHz}$.

- The Solar Orbiter Heliospheric Imager (SoloHI; Howard et al. 2020) will gather images of both the quasi-steady flow and transient disturbances in the solar wind over a FOV of $40^{\circ}$ by observing visible sunlight scattered by solar wind electrons.

- The Spectral Imaging of the Coronal Environment (SPICE; SPICE Consortium 2020), a European-led facility instrument, is an EUV imaging spectrograph designed to remotely characterize plasma properties of the solar corona.

- Spectrometer/Telescope for Imaging X-rays (STIX; Krucker et al. 2020) provides imaging spectroscopy of solar thermal and nonthermal X-ray emission from 4 to $150 \mathrm{keV}$. STIX will provide quantitative information on the timing, location, intensity, and spectra of accelerated electrons, and of high-temperature thermal plasmas mostly associated with flares and/or microflares.

- The Solar Wind Analyser (SWA; Owen et al. 2020) instrument suite comprises three sensors and a shared data-processing unit (DPU), and will characterize the solar wind at spacecraft location (between 0.28 and $1.02 \mathrm{AU}$ ).

The SO orbital profile involves two Venus encounters (in December 2020 and August 2021) followed by one Earth encounter (November 2021) to bring the spacecraft to an orbit with a closest perihelion of $0.28 \mathrm{AU}$ and an increasing orbital tilt, reaching 18 degrees in the nominal mission (reached first in March 2025), 25 degrees at the start of the extended mission (reached first in January 2027), and 33 degrees in the extended mission (reached first in July 2029).

The SO science goals rely on coordinated observations with multiple instruments (Müller et al. 2020). The in situ instruments (Walsh et al. 2020) operate continuously along the orbit beginning in cruise phase, while the baseline for the remote sensing instruments (Auchère et al. 2020) is to have three observing win- dows per orbit, each lasting 10 days, which are called remote sensing windows (RSWs). The RSWs will typically be centred around perihelia as well as the heliographic latitude northern and southern maxima. The operational constraints, including limited telemetry due to orbital characteristics, variable data latency (up to 180 days) due to large variations in spacecraft-Earth distance, and limited memory space on board, motivated the need for longterm planning of top-level science operations (Zouganelis et al. 2020). The mission science outcome depends on coordinated observations of unpredictable solar activity. The high-resolution science requires fine-pointing to a target and therefore a change in spacecraft pointing can occur three days before the observation takes place.

Both the PSP and SO nominal mission phases will be following the ascending phase of the solar cycle, as shown in Fig. 1, which illustrates the coronal appearance and solar wind characteristics over a full cycle. The upper images show typical coronal structures as well as polar plots of the solar wind speed as measured by Ulysses during cycle 23, whose sunspot number count is shown immediately below. The subsequent solar cycle, 24 , is plotted below cycle 23 , showing it has lower intensity but similar extent. If the coming solar cycle is not too different, PSP and SO will experience the cycle periods indicated by the continuous blue and orange lines in their nominal phase, with the extended phases (dotted line continuations) following the descending phase of cycle 25 .

\section{Synergies between SO and PSP}

\subsection{The SO-PSP Coordination Working Group}

Given the continuous change in orbital configurations and the complex PSP and SO concepts of operations, close coordination between the SO and PSP teams is critical. For this reason, the SO mission has established a working group (the SO-PSP Coordination Working Group; SPC-WG, hereafter). The SPCWG consists of the Project Scientists of the two missions, along with the instrument principle investigators (PIs) and their Project Scientists (and/or other designates), and members of the SO science operations team. The objectives of the group are: (1) to identify orbital configurations when unique science-science that extends the science objectives of the individual missioncan be achieved, including periods for instrument crosscalibration; (2) to ensure that spacecraft resources are available to accomplish the science; (3) to optimize the coordinated campaigns, including other assets (e.g., Bepi-Colombo); and (4) to facilitate the dissemination of the results and initiate joint $\mathrm{SO} / \mathrm{PSP}$ meetings. The relevant information is collected, and is publicly available, at a dedicated page on the SO Science Operation Center website ${ }^{1}$.

Both SO and PSP require long-term planning well in advance (e.g., SO science planning starts at least six months in advance Zouganelis et al. 2020) to ensure that adequate resources (including downlink stations) are available. Hence, the SO-PSP coordination discussions for a particular opportunity need to start more than six months in advance, particularly when the campaigns require participation from the remote-sensing instruments. During SO cruise phase (2020-2021), there are only four RSWs, which involve primarily instrument checkouts (Auchère et al. 2020). Therefore, the opportunities for PSPSO coordination are rather limited. Nevertheless, the SPC-WG will be discussing any such opportunities as they arise. Once

1 https://issues. cosmos.esa.int/solarorbiterwiki/ display/SOSP/SO-PSP+Coordination+WG 


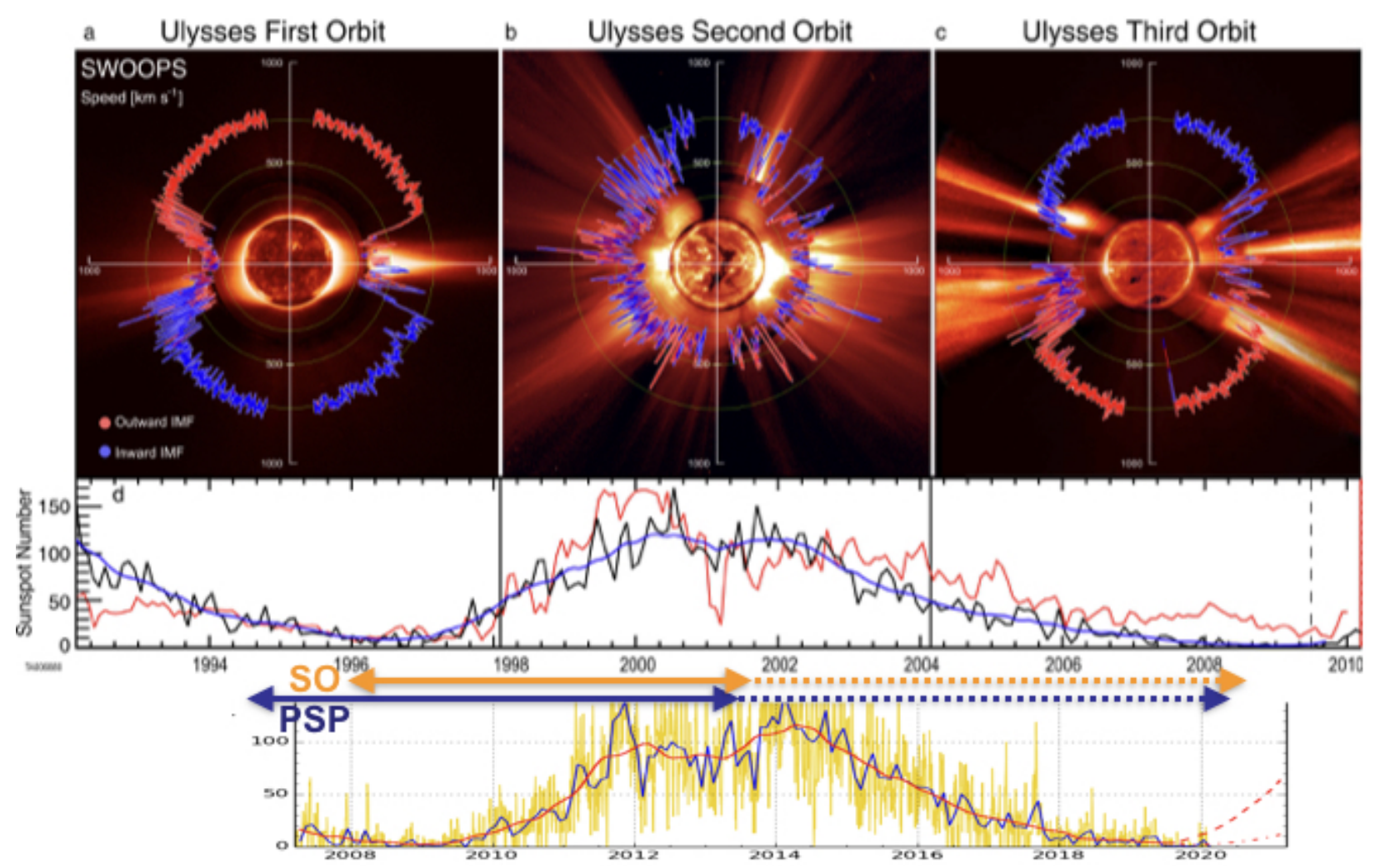

Fig. 1. Top: structure of the solar wind and changes in coronal appearance and solar wind characteristics over a full cycle, as observed by Ulysses and SOHO. Middle: corresponding sunspot number. Adapted from McComas et al. (2013). Bottom: sunspot numbers for cycle 24 as a proxy for the activity expected in Cycle 25 during the PSP and SO mission time lines (sunspot numbers have been corrected; Clette \& Lefèvre 2016).

SO enters the science phase of the mission in early 2020, the SPC-WG will start holding regular planning meetings in order to provide input into the mission-level science planning activities. These online meetings will be focused primarily on serving the SO science planning needs but we envision broader discussions. Specifically, the SPC-WG activities will be structured around:

- Science opportunities specific to SO: science goals to be addressed during a given long-term planning period (LTP) and optimal location of RS windows (typically three windows of 10 days).

- High-level planning of the whole six-month period, schedule of SO Observing Plans (SOOPs); see Zouganelis et al. (2020) for details.

- Simulation of resources and optimization of instrument observing programs.

- Opportunities for coordinated science or cross-calibration campaigns with other missions like Hinode, Solar Dynamics Observatory (SDO), STEREO, observatories like DKIST, the Swedish $1 \mathrm{~m}$ Solar Telescope SST, the Goode Solar Telescope GST, the New Vacuum Solar Telescope NVST, the Low Frequency Array LOFAR, Murchison Wide Field Array MWA, the Atacama Large Millimeter/submillimeter Array ALMA, and balloons such as Sunrise III (Solanki et al. 2017) and so on.

\subsection{Synergistic configurations of PSP and SO}

The differing instrumentation on PSP and SO, in addition to the differing orbits of the two spacecraft, provides a natural synergy which is further enhanced by the presence of other space- and ground-based assets along the Sun-Earth line. This is further added to by STEREO-A, whose remote sensing instrumentation provides a fourth potential line of sight through the solar corona and accelerating solar wind.

The complementarity of PSP and SO may be summarized in terms of relative orbital configurations, that is, specific periods of time where the Sun-PSP-SO angle is of specific interest, and in terms of contextual solar atmospheric measurements that SO may make via remote-sensing instrumentation. The latter are particularly relevant when PSP is in an encounter period on the far side of the Sun as seen from Earth but within the FOV of SO. In addition, the orbits have a series of conjunctions where PSP is closer to the Sun than SO - usually rapidly preceded and followed by quadratures in which PSP appears above the solar limb for the off-disk remote sensing instruments of SO, either in the ram or anti-ram direction. These configurations are labeled sequentially: E1, C1, Q1, etc. There are also a few conjunctions with SO inside the orbit of PSP, as well as quadratures with SO in the FOV of the single remote sensing PSP instrument suite, WISPR. Before describing the configurations in detail, we explain the meaning and significance of conjunctions and solar wind connectivity between spacecraft at different distances from the Sun.

Solar rotation implies the well-known fact that the magnetic field lines will take on a spiral shape (Parker 1958) starting from the radial distance at which corotation ceases even for a steady, homogeneous, and essentially radial solar wind outflow. This means that, within this approximation, two spacecraft that are contemporaneously aligned along the same spiral fieldline are sensing solar wind emitted from the same region on the solar surface, with the caveat that dynamical processes at the chromosphere-corona interface have not dramatically altered the solar footpoint within the time corresponding to the radial 
propagation from the measurement of one spacecraft to that of the other. In this case, one would expect a similar type of wind, that is, with the same composition, distribution function shapes, and turbulence characteristics, except for the intrinsic dynamical evolution associated with the travel time: composition and ionization state should however remain invariant, and it is here that the SO observations will make a fundamental contribution, given the limited measurements (alpha particles and energetic ions) that PSP can make of such quantities.

A different type of alignment occurs when two spacecraft at different radial distances separated by $\delta R$ cross the same spiral field line with a time difference corresponding to the travel time $\tau=\delta R / V_{\text {sw }}$ at the associated wind speed $V_{\text {sw }}$ from one spacecraft to the other. In this case, the two spacecraft would actually be traversing the exact same plasma parcel, changed only by ongoing dynamical processes occurring over time $\tau$. Tentative examples of the two types were provided for PSP encounter E1 in Panasenco et al. (2020). Again, composition and ionization states should remain unaltered during the transport, meaning that such measurements from SO would allow an essentially unambiguous determination of alignment in this case as well. At least theoretically, the spherical solar wind expansion aids in the alignment precision required to actually carry out the two types of spacecraft connection. A small time interval $\delta t$ of measurements at one spacecraft corresponding to a plasma parcel with an extension $\delta \alpha$ in the frame rotating with the Sun will, within the same approximations, maintain the same angular extent, corresponding to a linear extent along the spiral that increases in direct proportion to distance from the Sun.

Both types of alignment (which are typically separated by 1-3 days) are important in the unpredictable case of bursty events involving SEP emission and their acceleration. Changes in spectra and longitudinal distribution of SEPs should allow fundamental new insights into the relative role of source (generation, trapping, and escape time and the role of shocks vs. other seed particle generation mechanisms) versus propagation effects (field line random walk and diffusion property) in SEP distribution formation.

In addition to the encounters, because PSP crosses the co-rotation radius, which leads to retrograde motion as compared to the solar surface, there are two periods immediately before and after encounter, dubbed fast radial scans, where PSP runs towards and away from the Sun while maintaining almost the same longitude (in the final orbits, about 20 solar radii in extent over a period of a day or less). The presence of SO with its in situ and remote sensing observations could be planned, allowing us, in some instances, to fully determine the evolution of a plasma parcel from the outer corona out to at least the orbit of Mercury. A caveat for all of the above statements is related to the relative orbital inclination, of especially SO, which increases significantly beyond the cruise phase thereby reducing the scientific utility of such configurations.

The solar wind is not a homogeneous, spherically expanding medium, but instead, slow and fast streams coexist and may overlap or contain embedded intrinsically time-dependent outflows. Solar rotation means that different velocity streams and magnetic field shears may interact both compressively, as in the development of corotating interaction regions, and via KelvinHelmholtz-type linear and nonlinear instabilities that evolve with distance from the Sun. The former takes some time to develop on the largest scales, though smaller scale interactions could lead to shocks in the presence of large-scale equatorial extensions of polar coronal holes for example. The second type of interaction is clearly seen in the evolution of helmet streamer stalks and the
Table 1. PSP-SO configurations (Sect. 3.2).

\begin{tabular}{lcc}
\hline \hline Dates & PSP & SO \\
\hline $22-30 / 09 / 2020$ & E6 & C1, Q1, Q2 \\
$14-18 / 01 / 2021$ & E7 & Q3 \\
$24 / 04 / 2021-01 / 05 / 2021$ & E8 & C2, Q4, Q5 \\
$07 / 08 / 2021-20 / 08 / 2021$ & E9 & C3, Q6 \\
$18 / 09 / 2021$ & & C4 \\
$19-23 / 11 / 2021$ & E10 & C5, Q7 \\
$23-27 / 02 / 2022$ & E11 & C6, Q8, Q9 \\
$05 / 04 / 2022$ & & C7 \\
$29 / 05 / 2022-05 / 06 / 2022$ & E12 & C8, Q10 \\
$04-07 / 09 / 2022$ & E13 & C9, Q11, Q12 \\
$20 / 10 / 2022$ & & C10 \\
$09-12 / 12 / 2022$ & E14 & C11, Q13 \\
$16-20 / 03 / 2023$ & E15 & C12, Q14, Q15 \\
$17 / 04 / 2023$ & & C13 \\
$20-27 / 06 / 2023$ & E16 & C14, Q16 \\
$27 / 09 / 2023-05 / 10 / 2023$ & E17 & C15, Q17 \\
$12 / 10 / 2023$ & & C16 \\
$27-30 / 12 / 2023$ & E18,Q18 & \\
$20 / 03 / 2024-8 / 04 / 2024$ & E19 & C18, C19, Q19 \\
$28-30 / 06 / 2024$ & E20 & C20,Q20 \\
$25 / 09 / 2024-12 / 10 / 2024$ & E21 & Q21 \\
$22-25 / 12 / 2024$ & E22 & C21, Q22 \\
$20 / 03 / 2025-04 / 04 / 2025$ & E23 & C22, C23, Q23 \\
$17-22 / 06 / 2025$ & E24 & C24 \\
\hline & &
\end{tabular}

development of the heliospheric current sheet. In all cases, both in situ and remote sensing SO observations will be fundamental in discriminating solar wind source from solar wind evolutionary effects, allowing for a new understanding of coronal heating, solar wind acceleration, and stream evolution with distance from the Sun.

Movies of the SO (and PSP) trajectories are also available at the SO Science Operation Center website ${ }^{2}$. The reader is encouraged to examine them in conjunction with the discussion below.

In the remainder of this section, we give a "grand tour" of orbital configurations that are of interest for synergistic SO-'-PSP and other space- and ground-based assets. These configurations are ordered in time and labeled with several shorthand keys, as follows: "E": PSP orbit (Encounter) number, "C": conjunctions between PSP and SO (that most often have PSP between SO and the Sun, though interesting conjunctions with SO inside the PSP orbit are also called out), and "Q": quadratures, with PSP appearing above the solar limb for SO remotesensing instruments. Table 1 provides a summary of the different configurations discussed in the text below.

E6, C1, Q1, Q2, 22-30 September, 2020. This interval covers PSP's sixth solar encounter, and the first with a perihelion distance just inside $20 R_{s}$ from Sun-center. PSP-SO conjunction occurs at PSP perihelion on the 27 September, 2020. Throughout E6, SO will still be in its cruise phase (ending in November 2021), but because the Earth will be lagging behind PSP by between $90^{\circ}$ and $270^{\circ}$, and therefore the solar footpoint of PSP will be hidden from view from the Earth, any remote sensing measurements from SO would substantially help to understand the context of PSP measurements. Specifically, photospheric

2 https://issues.cosmos.esa.int/solarorbiterwiki/
display/SOSP/Orbit+Plots\%3A+February+2020+Trajectory 
magnetic field measurements from the SO/PHI suite would allow a study of PSP connectivity to the solar surface. Even a small number of other remote sensing measurements, from SPICE, EUVI, or METIS for example, would allow an initial test of how complementary measurements (e.g., composition) might aid in identifying solar regions of origin for the solar wind plasma. The in situ suites of SO should be turned on and taking measurements throughout the cruise phase allowing for measurements of the plasma, magnetic field, and composition. SOLOHI (located in the anti-ram direction) will have PSP in the FOV after E6. STEREO-A, on the opposite side of the Sun to E6 with respect to PSP, will see SOLOHI rise at the east limb and be in quadrature on 29 September 2020.

E7 and Q3, 14-18 January, 2021. During this interval, STEREO-A, Earth, and SO will provide $360^{\circ}$ coverage of the heliosphere, and just before E7, on 14 January, 2021, PSP will be in conjunction with STEREO-A, followed by conjunction with Earth on 16, E7 on 17, and quadrature Q3 on the east limb (trailing) with SO on 18 January, 2021. Synergies here arise mostly from potential remote-sensing measurements from SO and STEREO-A and from assets on the PSP-Earth line (SDO, Hinode) and ground-based instrumentation.

E8, C2, Q4, Q5, 24 April-1 May, 2021. Throughout this period $\mathrm{SO}$ will be in quadrature with Earth, appearing from Earth on the solar east limb. In rapid succession over E8, PSP will appear in quadrature from SO on the east limb (trailing), then in conjunction with SO at perihelion on 28 April, with PSP at a distance of $15.5 R_{S}$, then in conjunction with STEREO-A on 29 April and with Earth on 30 April. STEREO-A will be positioned trailing Earth by $45^{\circ}$ midway to $\mathrm{SO}$ in longitude. This PSP encounter is extremely favorable to a potential joint in situ-remote-sensing campaign comprising PSP, SO, STEREOA, SDO, Hinode, and ground-based instrumentation.

E9, Q6, C3, 7-20 August, 2021. The PSP begins encounter E9 by going through quadrature appearing from Earth above the Sun's west limb, followed by quadrature Q6 on the Sun's east limb as seen by SO at perihelion on 9 August, then in conjunction with SO coming out of perihelion on 12 August, then in conjunction with STEREO-A on the 20 August, 2021, while at about $0.3 \mathrm{AU}$.

C4, 18 September, $b$ 2021. This will be an inferior triple SO-PSP-STEREO-A conjunction, with SO inside the orbit of Venus around 0.6 AU, PSP at about 0.7 AU, and STEREO-A at 0.9 AU. This will be an occasion for joint in situ studies of the properties of turbulence and dynamics of a solar wind stream between 0.5 and $1 \mathrm{AU}$.

E10, C5, Q7, 19-23 November, 2021. As PSP approaches encounter E10 with perihelion on 21 November - its first at a distance of $13.3 R_{S}$ - it crosses conjunction with SO in encounter with Earth, before finding itself in quadrature on the west limb as seen from both Earth and SO at perhelion. This period may be of importance for magnetogram cross calibration between $\mathrm{SO} / \mathrm{PHI}$ and HMI on SDO, but also a good opportunity for spectroscopic and coronagraph measurements as well as in situ measurements from SO.

E11, C6, Q8, Q9 23-27 February, 2022. PSP approaches encounter E11 beginning with a trailing quadrature Q8 on 23 February, 2022, appearing on the east limb of the Sun from SO, before rapidly going through conjunction with STEREO-A and then SO and Earth (24 and 25 February, respectively) at perihelion with the Earth-Sun-STEREO-A angle $\simeq 40^{\circ}$ and the Earth-
Sun-SO angle decreasing from $\simeq 15^{\circ}$ to $0^{\circ}$ on 7 March, 2022, with SO at $0.5 \mathrm{AU}$. There will be clear opportunities here for cross calibration of magnetic field measurements, as well as a true stereoscopic reconstruction of the solar corona crossed by PSP, with opportunities for all the PSP-SO objectives.

C7, 5 April, 2022. The second inferior conjunction between SO and PSP with SO inside 0.5 AU and PSP approaching aphelion at $\simeq 0.7 \mathrm{AU}$.

E12, C8, Q10, 29 May-5 June, 2022. Approaching E12, PSP goes into conjunction C8 with SO on 30 May, then rapidly through quadrature Q10 and then into an opposite quadrature with STEREO-A, $\simeq 165^{\circ}$ from SO, as it crosses perihelion between 1 and 2 June, 2022. This will be an opportunity for reconstructing the $3 \mathrm{D}$ heliosphere given that Earth is still leading STEREO-A by about $40^{\circ}$.

E13, C9, Q11, Q12, 4-7 September, 2022. E13 is occulted for Earth and STEREO-A, but PSP crosses trailing quadrature (east solar limb) Q11 with respect to SO on 4 September, 2022, in conjunction with SO on 6 September (at perihelion) and opposite quadrature on 7 September. Remote-sensing observations from SO would be fundamental to unraveling the solar connection of PSP and following the source regions of the wind into the heliosphere.

C10, 20 October, 2022. 20 October, 2022, will see the third inferior conjunction between SO and PSP with SO at about $0.3 \mathrm{AU}$ and PSP approaching aphelion at $\simeq 0.7 \mathrm{AU}$.

E14, C11, Q13, 9-12 December, 2022. Going into perihelion, PSP goes into conjunction $\mathrm{C} 11$ with $\mathrm{SO}$, which is trailing Earth by only about $\simeq 30^{\circ}$ in longitude, on 9 December, 2022, then into conjunction with Earth on 10 December, and perihelion in quadrature Q13 on the solar west limb with SO on 11 December, followed by quadrature with Earth on 12 December, 2022.

E15, C12, Q14, Q15, 16-20 March, 2023. Going into perihelion, PSP goes into trailing quadrature with SO on 16 March, 2023, conjunction with SO right after perihelion on 17 March, followed by conjunction with Earth, that is leading SO still by only about $\simeq 30^{\circ}$ in longitude, on 18 March, and west limb quadrature with SO on 20 March, 2023. STEREO-A is sandwiched in longitude between SO and Earth, allowing comprehensive remote sensing and in situ joint studies of the corona.

C13, 17 April, 2023. The fourth SO-PSP inferior conjunction takes place on 17 April, 2023, with SO just coming out of perihelion close to $0.3 \mathrm{AU}$ and less than $7^{\circ}$ above the ecliptic and PSP close to aphelion around $0.7 \mathrm{AU}$.

E16, C14, Q16, 20-27 June, 2023. Going into perihelion, PSP goes into conjunction with SO on 20 June, 2023, right before perihelion and leading quadrature Q15 (west limb of the sun) on the 21 June, at which time PSP appears in quadrature above the east limb for Earth, followed by conjunctions with STEREO-A and Earth coming out of perihelion on 26 and 27 June, 2023. SO will be very close to the ecliptic.

E17, C15, Q17, 27 September-5 October, 2023. PSP approaches encounter E17, the first with a perihelion at $11.2 R_{S}$, The SO leads Earth by slightly more than $180^{\circ}$ and is approaching perihelion. PSP crosses leading quadrature with Earth and STEREO-A (now just leading Earth) just before perihelion on 27 September, when it crosses trailing quadrature Q with SO and then conjunction $\mathrm{C}$ on 28 September, 2023, with SO crossing 
perihelion on 5 October. This opportunity should not require special planning by $\mathrm{SO}$ at it is the perihelion remote sensing observing window; however, SO may be inclined too many degrees south of the ecliptic $\geq 10^{\circ}$ to make joint in situ alignments worthwhile.

C16, 12 October, 2023. Fifth SO-PSP inferior conjunction, with SO just coming out of perihelion close to $0.3 \mathrm{AU}$ and crossing the ecliptic, with PSP at about 0.5 AU. This will be an excellent opportunity for joint in situ measurements of the wind between 0.3 and $0.5 \mathrm{AU}$.

E18, C17, Q18, 27-30 December, 2023. As PSP approaches encounter E18, it is in conjunction $\mathrm{C} 17$ with $\mathrm{SO}$ on 27 December, 2023, with Earth and STEREO-A on 28 December, perihelion later on the same day, and leading quadrature Q17 with SO. The PSP solar footpoint will be visible throughout perohelion, a superb opportunity for synergistic studies on all objectives using all in situ and remote-sensing assets.

E19, C18, C19, Q19, 20 March-8 April, 2024. SO is in conjunction with Earth on 19-20 March, 2024, just outside of $0.4 \mathrm{AU}$, at the beginning of its southernmost remote-sensing window, and is then in conjunction with STSTEREO-A on 22 March, to be followed consecutively by its own perihelion (3 April) window. PSP is coming into perihelion in opposite conjunction with the Sun on 27 March, and is in trailing quadrature Q18 with SO at perihelion on 29 March. Observations of the solar magnetic field from Earth and SO allow excellent reconstruction of the magnetic field that PSP will be crossing at perihelion, and off limb observations from SO will provide additional measurements of coronal and solar wind properties. This encounter has the additional property that SO remotesensing and in situ measurements will be taken throughout the PSP encounter, and more precisely conjunction C18 will occur while PSP is in a fast radial scan as it moves out of the encounter. This provides the chance to reconstruct the detailed properties of a solar wind stream from $11.2 R_{S}(0.05 \mathrm{AU})$ all the way to 0.3 AU. On 7-8 April, the sixth SO-PSP inferior conjunction occurs at about $0.3 \mathrm{AU}$, right as $\mathrm{SO}$ exits its remote sensing perihelion window, providing a unique opportunity for multispacecraft inner heliospheric solar wind turbulence and solar wind property studies (though SO is $5-7^{\circ}$ north of the ecliptic).

E20, C20, Q20, 28-30 June, 2024. Between 28 and 30 June, 2024, PSP E20 is behind the Sun as seen from Earth, but $\mathrm{SO}$ is almost in opposite conjunction with Earth, leading by about $170^{\circ}$ in longitude. A combination of Earth and SO views of the photospheric magnetic field and corona would provide full $3 \mathrm{D}$ context essential to the PSP measurements during this perihelion, at the end of which PSP will be in quadrature for SO on the Sun's west limb. The PSP is then in conjunction with Earth coming away from the encounter on July 5, 2024.

E21, Q21, 25 September-12 October, 2024. E21 is less than optimal as the PSP perihelion occurs behind the Sun both as seen from SO and from Earth (and off the Sun's west limb as seen by STEREO-A). PSP is in conjunction with STEREO-A on its way in, on 27 September, 2024. However, the SO remote sensing south and then perihelion windows are open from 15 September through 4 October, allowing the collection of far-side magnetic field and plasma data. This provides fundamental context for the PSP perihelion traverse of the corona on 30 September, while in quadrature off the Sun's east limb as seen by SO, which is at its own perihelion on that same day. PSP and SO remain fairly close in longitude for a few more days.
E22, C21, Q22, 22-25 December, 2024. On 22 December, 2024, the first PSP perihelion at $9.86 R_{S}$ occurs on disk as seen from SO (trailing Earth by about $15^{\circ}$ in longitude), Earth, and STEREO-A. PSP is in conjunction first with SO and then with Earth and STEREO-A, all on the same day as it rapidly crosses the solar disk into perihelion. On 23 December, perihelion occurs behind the Sun both as seen from SO and from Earth from 23 to 24 December, and is in view in quadrature on the west limb of the Sun throughout 24 December by SO on its way out. Outside the SO remote sensing windows, this is an important opportunity for a specific additional request: proximity to Earth, allowing for sustained data transfers.

E23, C22, C23, Q23, 20 March - 4 April, 2025. The second perihelion at $9.86 R_{S}$ on $20 \mathrm{March}, 2025$, has PSP coming around the east limb of the Sun as seen from Earth, SO, and STEREOA. The entire encounter occurs during the south remote sensing window for SO, with PSP in quadrature on the east limb of the Sun on the 22 March at perihelion, and in conjunction on 23 March, 2025. SO is coming out of conjunction with the Earth (13 March) is in conjunction with STEREO-A on 22 March, and is itself in perihelion on 1 April, followed by inferior conjunction with PSP on 3 April while completing its perihelion remote sensing window. This configuration lends itself to synergistic studies for all PSP and SO scientific objectives (the latitudinal separation of the SO and PSP is large at PSP perihelion, over $10^{\circ}$, but smaller $\simeq 5^{\circ}$ at SO's inferior conjunction.

E24, C24, 17-22 June, 2025. The final nominal mission perihelion for PSP, E24, sees SO and the Earth in quasi-opposite conjunction, spanning $170^{\circ}$, with PSP in conjunction with SO on 18 June, 2025, and perihelion and quadrature for both SO (on the west limb of the Sun) and Earth (on the east limb) on 19 June. This window is less than optimal as the SO north remote sensing window will have closed over a month earlier, but it would be opportune to schedule an extra observing campaign for SO given that the PSP perihelion is also hidden from view from STEREO-A.

\section{Science questions for PSP-SO}

The following are two examples of science questions where PSP-SO synergies may provide fundamental insights. Detailed science questions are discussed for PSP in Fox et al. (2016) and for SO in the detailed science plan (Zouganelis et al. 2020).

\subsection{Origin of steady and nonsteady slow solar wind}

While it has been recognized since the Skylab era of the 1970 s that high-speed solar wind emanates from large coronal holes, the origin of wind with near-Earth speeds in the range 300$450 \mathrm{kms}^{-1}$ remains at least partially unknown (Abbo et al. 2016). One hypothesis is that the slow wind simply originates from more rapidly expanding coronal hole boundaries (Wang \& Sheeley 1992; Arge \& Pizzo 2000). This is suggested by the inverse correlation between wind speed and the rate at which the underlying flux tube expands in the corona, as inferred from outward extrapolations of the measured photospheric field. This may be understood physically if the coronal heating rate is taken to depend on the local magnetic field strength. The effect of rapid flux-tube divergence is then to concentrate the heating near the coronal base, thereby increasing the mass flux but decreasing the energy per proton available to accelerate the wind; moreover, with the temperature maximum being located at lower heights, the freezing-in temperatures will be higher in slow wind than in 
fast wind, as suggested by observations (Wang \& Sheeley 2003; Cranmer et al. 2007).

However, a distinction should be made between the boundary regions of large-scale polar coronal holes and rapidly expanding narrow coronal hole extensions or narrow, widely expanding equatorial coronal holes. While semi-empirical, fully self-consistent stationary models of solar wind acceleration along a prescribed open magnetic field explain this effect in terms of the appearance of a bifurcation, at a specific heliomagnetic latitude in the location where the solar wind critical point occurs, these also lead to an accelerating, decelerating, and re-accelerating flow profile along the more rapidly expanding field lines with a strong latitudinal pressure gradient (Lionello et al. 2014) leading to a back-reaction and a more homogeneously expanding coronal hole.

On the other hand, narrow holes expanding above active regions or between pseudostreamers could support a slow wind solution (Panasenco et al. 2019). The existence of Alfvènic slow wind, that is, slow wind streams containing the typically fast-wind turbulence made up of outwardly propagating Alfvèn waves (see Panasenco et al. 2020 and references therein), may be confirmation of the latter idea.

That at least a small fraction of the slow wind comes from closed-field regions is indicated by white-light observations of streamer blobs and ray-like fine structure in the immediate vicinity of the heliospheric current sheet (HCS). Distinctive compositional properties of the slow solar wind (Geiss et al. 1995; von Steiger et al. 2000) also suggest a different origin of this plasma outside coronal holes. However, the filling factor of the slow wind in the heliosphere seems to be too large to arise only from the helmet streamer cusps, and so magnetic field and plasma transport, and instabilities (Rappazzo et al. 2005), involving processes at coronal hole boundaries and in the quiet sun may be involved. Coronal holes appear to rotate as a solid body, not being destroyed by the meridional shear in the solar photospheric rotation rate. The quasi-rigid rotation of coronal holes then requires continual footpoint exchanges between open and closed field lines, with the reconnection taking place at the streamer cusps or below (Fisk 1996).

Other slow wind sources might be small, temporarily open regions interacting with closed corona, transient coronal holes neighboring active regions (Neugebauer \& Liewer 2003), or more highly complex transport associated with the fractal meandering of coronal hole boundaries. The streamer structure in the outer corona consists not only of helmet streamers, which are here defined as separating coronal holes of opposite polarity, but also of pseudostreamers, which separate coronal holes of the same polarity (Riley \& Luhmann 2012; Panasenco et al. 2013, 2019; Abbo et al. 2016). While lacking current sheets, pseudostreamers have plasma sheet extensions that outline a network of separatrices branching off from the HCS. These sheets again consist of ray-like features that may be a product of interchange reconnection at the underlying X-point, which typically lies below $1 R_{s}$. The adjacent open flux undergoes a nonmonotonic expansion, diverging rapidly between the coronal base and the X-point but then reconverging. According to the "S-web" model of Antiochos et al. (2011), interchange reconnection gives rise to wide bands of slow wind that surround not only the HCS but also the pseudostreamer separatrices. Identifying pseudostreamer stalks in the solar wind, and the eventual transient plasma release along them, is one of the goals in determining the origin of the slow wind.

\subsection{Energetic particle acceleration, transport, and distribution.}

There are several mechanisms that produce energetic particles originating from the solar corona and spreading out into interplanetary space, leading to distributions that eventually fill the heliosphere. Each of these mechanisms leaves its imprint on the flux properties (temporal variations, abundances, spectra, and directional information) of the energetic particle events they generate. However, once the particles are released from their sources, they propagate through the solar wind and interplanetary magnetic field which are pervaded by turbulent fluctuations. These may act as scattering centers for the particles that will also follow the turbulent meandering of the field lines themselves. Helios observations (Wibberenz \& Cane 2006) showed that SEP events close to the Sun are much less disturbed by interplanetary transport effects compared to $1 \mathrm{AU}$ observations. As the observing spacecraft goes farther from the Sun, the imprints of the above-mentioned energetic particle fluxes get blurred, making identification of the acceleration process(es) much more difficult. The periods when PSP and SO are connected through the same interplanetary magnetic field lines provide a unique opportunity to unveil the transport phenomena at work in the inner heliosphere and, eventually, they will be crucial to identifying the SEP injection, acceleration, trapping, and release processes. By mapping the energetic particle population at different heliolongitudes in the inner heliosphere, EPD and IS $\odot$ IS measurements will shed light on circumsolar energetic particle events (Gómez-Herrero et al. 2015) whose origin remains unknown. Several mechanisms have been suggested, but the lack of inner heliospheric measurements at different helio-longitudes has so far prevented the root cause from being identified.

\section{Earth-orbiting and other space assets}

From the Earth's vantage point, the Solar Dynamics Observatory (SDO), Hinode, and IRIS all carry out extensive investigations of the solar atmosphere, from the solar photosphere out to about 1.2 solar radii. The SDO provides continuous observations of the Sun's photospheric magnetic field and EUV imaging at high cadence. These data will be key for providing global magnetic field modeling (along with the ground-based Global Oscillations Network, GONG; Harvey et al. 1987). In addition, the global views in the different wavelengths will provide opportunities for stereoscopy and provide key context images. An important co-observation is that by $\mathrm{SO} / \mathrm{PHI}$ on the one hand and SDO/MDI or GONG on the other; together these instruments will be able to better identify oscillation modes, probe the flows in the deep convection zone, and peer beneath the surface also under the poles. These joint observations will play an important role in answering the fourth SO top-level science question regarding the solar dynamo and the connections between the Sun and the heliosphere. In the following two sections, we describe further space missions that have small-field-of-view instruments and hence require more detailed forward planning.

\subsection{Hinode}

The ISAS/JAXA Hinode mission (Kosugi et al. 2007), previously known as Solar-B, was launched successfully in September 2006 from the Uchinoura Space Center in Japan using an $\mathrm{M}-\mathrm{V}$ launch vehicle. Hinode is designed to answer the fundamental question of how magnetic fields are formed and interact with the atmosphere to create the activity that we observe on the Sun. This ultimately addresses all phenomena 
that have an impact on the Sun-Earth system, such as the formation of the solar winds (both slow and fast), triggering of flares and coronal mass ejections, and formation and maintenance of filaments and prominences. There are three instruments on-board Hinode: the Solar Optical Telescope (SOT: Tsuneta et al. 2008), the X-ray Telescope (XRT: Golub et al. 2007), and the EUV Imaging Spectrometer (EIS: Culhane et al. 2007). Each of these instruments measures a critical part of the Sun's atmosphere, from the surface (photosphere) to the chromosphere, the transition region, and finally the outer and hottest part of the atmosphere, the corona. It is anticipated, barring any unexpected events, that the mission will continue to operate until the mid2020s. This provides significant overlap with the science phase of SO. Hinode provides unique data that are highly complementary to SO observations: it is the only mission that can obtain coronal spectral data, potentially providing "3D" coronal spectroscopy (including velocities and abundances) working alongside SO-SPICE; it is the only mission that provides soft X-ray imaging, giving unique views of eruptions; and it is the only mission to have continuous high-resolution spectro-polarimetric coverage close to 24 hours per day.

Hinode began providing complementary observations with PSP during its first perihelion in November 2018. It did this through the Hinode Observing Programme (HOP) that coordinates the three instrument observing modes with other facilities. The first one of these, designed for connection science with PSP, was HOP 366, the details of which are available online ${ }^{3}$. The goal was to observe the solar sources of the solar wind measured by PSP and others. To do this, global magnetic field modeling was used to predict the source of the solar wind on the disk. The small-field-of-view instruments were then pointed at this region. This is providing excellent experience for observing with SO. One of SO's main remote-sensing goals will be to observe the sources of the solar wind that will later be detected by the spacecraft in situ. This involves making use of the most up-todate magnetic connectivity modeling, which has been designed to connect the 'footpoints' of the solar wind sources that reach the spacecraft. Hinode will observe the sources of the solar wind, measuring the magnetic fields and plasma parameters that can link to the in situ data and track eruptions.

The different viewpoints between the two spacecraft will provide different stereoscopy opportunities, including, for the first time, photospheric magnetic field, which will provide tight constraints on the magnetic field and open new fields in coronal spectroscopy with the 3D information. To date, Hinode has provided the most accurate long-term view of the magnetic field at the solar poles. As SO slowly moves out of the ecliptic, the polar fields will be revealed with higher and higher accuracy. In 2025 for example, SO will have reached close to 20 degrees out of the ecliptic plane. This new information, combined at that point with close to two solar cycles of polar observations from Hinode, will be invaluable.

\subsection{The Interface Region Imaging Spectrograph}

The Interface Region Imaging Spectrograph (IRIS; De Pontieu et al. 2014) is a NASA Small Explorer Mission that was launched in 2013. It is an imaging spectrometer with slit-jaw imaging that probes the chromosphere and specifically explores solar chromosopheric dynamics in order to determine how energy flows through this region. The chromosphere is fundamental to

\footnotetext{
3 http://www.isas.jaxa.jp/home/solar/hinode_op/hop. php?hop $=0366$
}

understanding what causes the ejection of solar material: from the steady stream of the solar wind, to larger, explosive eruptions such as CMEs. IRIS contributes to the HOPs for PSP and will do so for SO. There are different instrument modes depending on both the solar wind source highlighted through the magnetic field modeling (coronal hole, active region) and the observed region (on-disk or at the limb) and there are high- and low-cadence observing modes for each option.

\subsection{Coronal and inner heliosphere coverage}

With its suite of instruments ranging from EUV to heliospheric imagers, the STEREO mission covers the Sun-Earth line comprehensively, from the inner corona to $1 \mathrm{AU}$, and consequently its synoptic program will provide context imaging for almost every point of the SO and PSP orbits and a third viewpoint for 3D reconstructions of solar transients. The LASCO coronograph is still in operation aboard the SOHO mission, providing yet another viewpoint with excellent coverage of the corona to $30 R_{S}$ for all PSP perihelia. In addition, STEREO has executed special campaigns for each PSP perihelion, involving high-cadence coronagraphic and/or EUV or heliospheric imaging, depending on the science target and the relative spacecraft configuration. The details are on a dedicated STEREO operations page ${ }^{4}$. Several of PSP's first results papers relied on STEREO observations (see Sect. 7) demonstrating the power of coordinated planning. The STEREO contributions included tracing solar structures from the Sun over the PSP and multi-viewpoint reconstructions of CMEs. The STEREO campaigns almost always involve special support from LASCO and occasionally from other assets, such as the Sun Watcher with Active Pixels (SWAP; Seaton et al. 2013). A similar approach is envisioned for SO RSW support. The SPC-WG includes members of the STEREO science team, and so a close and efficient coordination is ensured.

Coronal and heliospheric studies with be further augmented by the anticipated launches of the ESA Proba-3 (Zhukov 2019) satellites in 2022 and the Polarimeter to UNify the Corona and heliosphere (PUNCH; DeForest et al. 2019) in 2023. Proba-3 will obtain images of the inner corona $\left(1.08-3 R_{S}\right)$ but only intermittently $\left(\sim 12\right.$ hours week $\left.{ }^{-1}\right)$. However, this is a key region for assessing the origins of the solar wind, as discussed earlier, and the observations may be of considerable value, meaning that careful coordination between Proba-3 and SO/PSP is essential. PUNCH will provide continuous coverage (bar a short eclipse period every year, similarly to IRIS and Hinode) around the Sun from about $6 R_{S}$ to $1 \mathrm{AU}$ for a sun-synchronous orbit. Because of the FOV coverage, such observations will be highly complementary to both PSP/WISPR and SO/SoloHI.

\section{Ground-based instrumentation, PSP, and SO}

There is an extensive array of ground-based solar telescopes that stand ready to participate in coordinated campaigns with SO and PSP. An idea of the enthusiasm of the ground-based community can be gleaned from the highlights ${ }^{5}$ from the campaign for the fourth PSP perihelion in January 2020. This was a campaign under the Whole heliosphere and Planetary Interactions

\footnotetext{
${ }^{4}$ https://stereo-ssc.nascom.nasa.gov/plans/psp_ support/

5 https://whpi.hao.ucar.edu/whpi_ campaign-cr2226-highlights.php
} 
(WHPI) international initiative to study the solar minimum Sun and indeed a rehearsal for future PSP (and SO) campaigns.

\subsection{Radio observations}

Radio observatories are a particularly active section of groundbased support as they can observe both the disk and the extended corona and heliosphere. LOFAR in particular has allocated 1000 hours for PSP support and participated in the third and fourth PSP perihelia campaigns and several initial results are under review already. The Very Large Array (VLA), MWA, the Interplanetaery Scintillation (IPS) community, and several other radio observatories are adjusting their observing and maintenance plans around PSP (for now) and SO (before long) perihelia. As the activity (and solar radio emissions) picks up, radio observations will play an increasingly important role in deciphering PSP and SO observations. The IPS measurements have been providing and will continue to provide key heliospheric observations of density fluctuations and possible Faraday Rotation measurements through CMEs, in support of the white-light imaging from $\mathrm{PSP} / \mathrm{SO}$.

Another telescope that will be of interest to compare in particular with SO is ALMA, which is also capable of observing the Sun (Shimojo et al. 2017; White et al. 2017) and is attacking a whole range of important solar questions (see Wedemeyer et al. 2016). Joint observations with IRIS and SO will allow us to probe the solar chromosphere, the key interaction region between the photosphere and corona, more deeply than hitherto possible.

\subsection{The Daniel K. Inouye Solar Telescope}

In the visible/infrared regime, the main addition to our observing arsenal will be the Daniel K. Inouye Solar Telescope (DKIST), a $4 \mathrm{~m}$ solar telescope located on Maui, Hawaii, ground-breaking in its design and new instrumentation, which will probe fundamental processes on the Sun. DKIST will provide polarimetry of structures of about $20-30 \mathrm{~km}$ in size over a limited FOV depending on the instrument (and up to $~ 300$ arcseconds) from the visible to near- and thermal infrared. First light instruments include: the Visible Broadband Imager (VBI), the Visible Spectro-Polarimeter (ViSP), the Diffraction-Limited NearInfrared Spectropolarimeter (DL-NIRSP), the Cryogenic NearInfrared Spectropolarimeter (Cryo-NIRSP), and the Visible Tunable Filter (VTF).

- The VBI will be able to capture high-cadence images simultaneously in two spectral bands (blue and red): 390$550 \mathrm{~nm}$ (VBI blue channel) and 600-860 nm (VBI red channel). The optical FOV is 120 arcseconds and the physical FOV will be limited by the size of the CCD camera (about 45 and $69 \operatorname{arcsec}^{2}$ for the blue and red channels, respectively). The instrument can produce a mosaic by moving the detector to effectively cover the optical FOV. The spatial resolution provided by this instrument is 0.022 arcsec at $430.5 \mathrm{~nm}$ (blue) and 0.034 arcsec at $656.3 \mathrm{~nm}$ (red). A cadence of $3.2 \mathrm{~s}$ for reconstructed individual images at a single wavelength per channel is possible.

- ViSP will provide simultaneous precision measurements of all four Stokes parameters $(I, Q, U$, and $V)$ at three wavelengths in the visible spectrum. Its spectral resolution of nearly 200000 allows the profiles of spectral lines originating in the solar atmosphere to be almost fully resolved. Such measurements provide quantitative diagnostics of the magnetic field vector as a function of height in the solar atmosphere, along with the associated variation of the thermodynamic properties. Fur- thermore, information about protons and electrons in flares can be deduced from analyzing the polarization of strong lines during these events. The wavelength range is $380-900 \mathrm{~nm}$. The spatial resolution depends on the channel and reaches 0.07 arc$\mathrm{sec}$ at $630 \mathrm{~nm}$ with a variable FOV perpendicular to the slit and of 70 arcsec along the slit. ViSP can operate simultaneously with VBI, DL-NIRSP, and VTF, as long as the instruments observe different spectral bands.

- DL-NIRSP is designed to study solar magnetic fields at high spatial resolution with high spectral resolution and polarimetric sensitivity. There is simultaneous spectral coverage over three spectral windows, one in the visible and two in the IR. This instrument will carry out polarimetry, either Full Stokes polarimetry $(I, Q, U, V)$ or intensity only (this mode is also possible for ViSP). The FOV can be up to $120 \times 120$ arcsec. As before, it operates simultaneously with other instruments, except Cryo-NIRSP, and shares with them the adaptive-optics-corrected beam.

- The VTF is a Fabry-Perot based instrument designed to spectrally isolate narrow-band images of the sun at the highest possible spatial and temporal resolution from the DKIST telescope. The instrument allows the user to obtain 2D spectral data, Doppler velocity maps, transverse flows, and maps of the magnetic field components on spatial scales between $20 \mathrm{~km}$ (resolution) and $40000 \mathrm{~km}$ (FOV). The spectral range is from $520 \mathrm{~nm}$ to $870 \mathrm{~nm}$ and a spatial sampling of $0.014 \mathrm{arcsec}_{\mathrm{pixel}}{ }^{-1}$.

- Cryo-NIRSP will study the solar corona, including its magnetic fields, over a large FOV at near- and thermal-infrared wavelengths. Cryo-NIRSP will measure the full polarization state (Stokes $I, Q, U$ and $V$ ) of spectral lines originating on the sun at wavelengths from $1000 \mathrm{~nm}$ to $5000 \mathrm{~nm}$. It is the principal DKIST instrument capable of sensitively imaging the relatively faint infrared corona and the thermal-infrared solar spectrum up to half a solar radius above the limb. Cryo-NIRSP depends on the full coronagraphic capabilities of DKIST to observe both the near-limb (using DKIST's Lyot stops and a secondary focus occulting disk) and the more distant corona. Its thermal-infrared capabilities allow sensitive study of the solar disk in the CO lines. Near-limb capabilities allow unique observations of spicules, prominences, flares, and eruptive events in the low corona. The spatial sampling is $0.5 \operatorname{arcsec~pixel}^{-1}$ size for coronal observations, and 0.15 arcsec pixel $^{-1}$ sampling for disk observations (at 4.7 micron). The is, in coronal mode, 4 arc minutes parallel to the limb and 3 arc minutes perpendicular to the limb for near-limb observations and 5 arc minutes for far limb modes. Cryo-NIRSP works as a stand-alone instrument. By using the spectroscopic observations in conjunction with the instrument's context imager, density line ratios of the corona can be obtained.

DKIST science operations are scheduled to start in late 2020, just in time for joint observations with the SO mission, already in its cruise phase. The suite of DKIST instruments is versatile enough to address a variety of science questions. The set of most critical science goals for the first two years of operations, the DKIST Critical Science Plan, is being brought up through a process that includes a series of coordination workshops held during 2017/2018. One of these workshops was centered around bringing together science of SO, PSP, and DKIST. Details of these can be found in the Critical Science Plan ${ }^{6}$. The workshop helped identify three main areas of scientific interest when combining these facilities, which utilize the multiple viewpoints along with highly complementary observations:

6 https://wwW.nso.edu/telescopes/dkist/workshop-4/ 


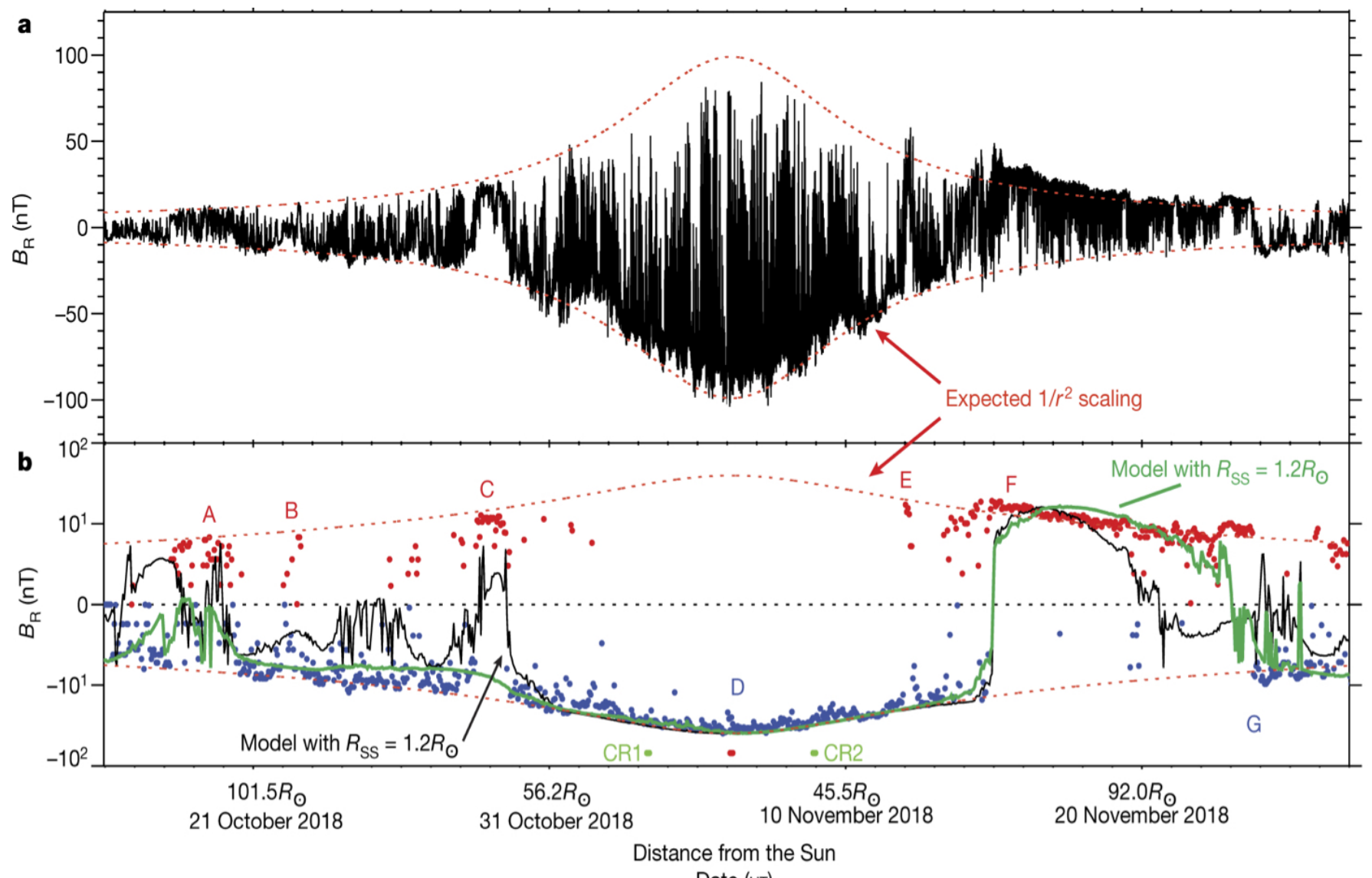

Fig. 2. Panel $a$ : PSP measured radial magnetic field BR over the first PSP encounter. The magnitude scales approximately as 1/r2 (red dotted lines; also in panel $\mathrm{b}$. We highlight the rapid large-amplitude polarity reversals $(\delta \mathrm{BR} /|\mathrm{B}|$ of the order of 1$)$. Panel $b$ : one-hour statistical modes of $\mathrm{BR}$ (bisymmetric logarithmic plot) show the large-scale radial field colored for polarity (red, outward; blue, inward). Predicted radial-field profiles from a PFSS model are shown in green and black (SS at 2.0 and 1.2 RS respectively). Adapted from Bale et al. (2019).

Stereoscopy. Having two facilities with different lines of sight opens up new possibilities for scientific exploration. These include observation of the magnetic field from different perspectives to constrain the magnetic field extrapolations. These are heavily relied upon to understand the morphology of the magnetic field that creates the solar wind and activity on the Sun. Reconstructing the 3D magnetic field will provide new insights into all of the structures on the Sun; particularly complex ones such as prominences. The quadrature situation will also allow us to explore the impact of averaging over optically thin plasma with DKIST while observing the regions participating included in the average from above with SO. In rarer cases when eruptions or flares are observed, the first high-resolution observations of current sheets from two complementary perspectives may be uncovered.

Polar Science. A key aspect of this collaboration is probing the polar magnetic fields, although the best opportunities for such investigations will occur later on in the SO mission. The polar fields are a key source of fast solar wind, and also a probe of the global magnetic fields of the Sun. The polar fields are currently poorly understood, and SO will provide the first ever meaningful view with SO/PHI. Alongside this, there is the well-known $180^{\circ}$ azimuth ambiguity that impacts Zeeman measurements. Stereoscopic observations will solve this problem, providing us with the first physical understanding of the polar fields, and how this creates the fast solar wind. The imaging and spectroscopy on SO will provide an understanding of the sources of wind in the corona, and their abundances, which will then be measured by the in situ instruments. High-resolution, high-sensitivity observations with DKIST would also complement SO long-term observations of the evolution of the polar solar magnetic field.

Solar wind. There are many opportunities for solar wind science with these two facilities. Stereoscopic observations are one obvious aspect to be exploited, but the coronal capabilities of DKIST instruments are also a great asset. An example is the search for the source of solar wind related to active regions. Using quadrature, DKIST could measure the coronal magnetic field of an active region at the limb with DKIST and simultaneously measure the active region head-on from SO. This will allow measurements of the photospheric magnetic field from SO, and the coronal field from DKIST. Spectroscopy and imaging from EUI and SPICE on SO will be used to measure the plasma parameters in the atmosphere that create part of the slow wind. The magnetic field topology in the corona measured by DKIST can be linked to the properties of the solar wind (e.g., He abundance, outflow velocity) measured by Metis and EUI. This will allow the solar wind expansion to be measured linking straight into the in situ measurements of the solar wind. Cryo-NIRSP observations can also be used to map chemical abundances in the corona.

The opportunities for excellent combined science are extensive. Details of the cases are presented on the DKIST Critical Science Plan website available from the Workshop link provided above. Community involvement is highly encouraged by 
contacting DKISTCSP@nso.edu. A white paper further describes the scientific possibilities of using DKIST, PSP, and SO as a multi-messenger constellation (Martinez Pillet et al. 2020).

\subsection{Other ground-based and balloon-borne telescopes}

In addition to DKIST, there are a number of further optical telescopes with apertures of $1 \mathrm{~m}$ or more. These include the $1 \mathrm{~m}$ Swedish Solar Telescope (SST; Scharmer et al. 2003) on the Canary Island of La Palma, the $1.6 \mathrm{~m}$ Goode Solar Telescope (GST), previously called the New Solar Telescope (NST; Cao et al. 2010) at Big Bear Solar Observatory, the $1.45 \mathrm{~m}$ Gregor telescope (Schmidt et al. 2012) on the Canary Island of Tenerife, and the New Vacuum Solar Telescope (NVST; Liu et al. 2014) by Fuxian lake in China. These telescopes complement DKIST in two ways: (1) Being distributed around the Earth in longitude, they can also observe at times DKIST cannot. Therefore, they will provide much more complete coverage. (2) They also have instruments with capabilities that complement those on DKIST, meaning that they can provide additional information to that coming from DKIST.

The SUNRISE balloon-borne solar observatory, which has successfully flown twice (Solanki et al. 2010, 2017), will fly again in 2022. By observing over $90 \%$ of the Earth's atmosphere from above, it will provide high-resolution, seeing-free data of unchanging quality (magnetograms, UV and IR spectropolarimetry), which can be used in conjunction with those obtained by instruments such as EUI, SO/PHI, and SPICE on SO. Sunrise will provide an excellent complement to the instruments on Solar SO for magnetic field disambiguation and stereoscopy of the solar photosphere.

\section{Early PSP observations}

Perhaps the first dramatic observation made by PSP during its first orbit was the persistent presence of extremely large-amplitude oscillations in the radial magnetic field, as illustrated in the top panel of Fig. 2 (Bale et al. 2019). In the figure, the dotted red line provides the expected $1 / R^{2}$ dependence of the field throughout E1, with a perihelion distance from the Sun of $35.7 R_{s}$. These reversals of the radial magnetic field, specifically between the times/distances marked $\mathrm{C}$ and $\mathrm{F}$ in red in the lower panel, did not correspond to heliospheric current sheet crossings, as demonstrated for example by the permanence of the electron pitch angle. In other words, PSP was not connected to a negative polarity region on the Sun, but rapid folds in the field, called switchbacks, were seen on timescales going from periods of seconds to more than an hour. An analysis of the corresponding velocity field shows that the fluctuations in radial velocity, $\delta V_{R}$, are well correlated to those of the magnetic field, with a correlation with $\delta B_{R}$ corresponding to outwardly propagating Alfvén waves. In addition, the magnitude of the total magnetic field remains almost constant, that is, the compressibility of the fluctuations is very small. The magnetic (and velocity, after subtraction of an appropriate average, Matteini et al. 2019) field vectors therefore rotate with the vector tip trajectories on a sphere, that is, switchbacks are spherical-arc, polarized, large-amplitude Alfvèn waves. One of the intriguing properties of such waves is that, in the presence of a magnetic field with an s-shaped backwards fold, the radial component of the velocity always presents a positive enhancement, or radial jet (Matteini et al. 2019). Remarkably, the wind seen by PSP displayed a strongly dominant outward-propagating spectrum of Alfvénic fluctuations throughout the encounter, even though the velocity of the solar wind was between 250 and $400 \mathrm{~km} \mathrm{~s}^{-1}$ for most of the encounter, a feature that is rarely seen in slow solar wind streams at Earth and beyond.

A second, surprising PSP observation was the presence of a strong tangential solar wind flow component, once PSP dropped below a quarter of the distance from the Sun to the Earth, peaking at up to $40-50 \mathrm{~km} \mathrm{~s}^{-1}$ at E1 and E2 (Kasper et al. 2019). This was indirectly confirmed by WISPR imaging observations showing very little relative speed between the observed whitelight rays and streamers, and the spacecraft (Howard et al. 2019). The rotational velocity being up to an order of magnitude higher than expected on the basis of simple models, this finding is all the more suprising because even if compatible with an extended magnetically enforced coronal corotation out to the Alfvén radius (where the wind speed overtakes the Alfvén wave speed), it seems to arise during the second-weakest solar cycle of the space age, that is, in the presence of extremely weak coronal magnetic fields.

From the energetic particle point of view, early IS $\odot$ IS observations (McComas et al. 2019) have shown the existence of energetic particle events accelerated both locally and remotely. These include acceleration at stream interaction regions, where energetic particles are observed more locally than at $1 \mathrm{AU}$, as well as lower energy impulsive events than previously seen driven by acceleration near the Sun, and also energetic particles associated with CMEs.

\section{Conclusions}

The heliophysics community is excited to have SO and PSP concurrently operating in the inner heliosphere loaded with highly capable and highly sophisticated payloads. In this short report, we attempt to outline the enormous potential for scientific discovery arising from the synergistic observations from PSP, SO, and a slew of other space- and ground-based observatories.

Joint measurements during PSP-SO alignments, complemented by alignments with Earth and dual PSP-Earth and SO-Earth, as well as alignments with STEREO-A, SOHO/ LASCO, and BepiColombo will allow a better understanding of the in situ evolution of solar wind plasma flows as well as the full three-dimensional distribution of the solar wind from a purely observational point of view. Spectroscopic observations of the corona, optical and radio observations, and direct in situ observations of the accelerating solar wind combined will provide a new foundation for understanding the fundamental physical processes leading to the energy transformations from solar photospheric flows and magnetic fields into the hot coronal plasma and magnetic fields and finally into the bulk kinetic energy of the solar wind and SEPs. Initial PSP observations already provide a compelling rational for new measurement campaigns from SO as well as with ground- and space-based assets, and highlight the need for careful advance planning to optimize the science return from this unprecedented opportunity to study and understand the workings of the environment that surrounds our planet.

Acknowledgements. M.V. was supported by the NASA Parker Solar Probe Observatory Scientist Grant No. NNX15AF34G as well as the SOLOHI Phase E program at JPL. A.V. contribution was supported by the WISPR Phase E program at APL and NASA 80NSSC19K1261 Grant. J. Rodríguez-Pacheco thanks FEDER/MCIU-AEEI/Proyecto ESP2017-88436-R. Parker Solar Probe was designed, built, and is now operated by the Johns Hopkins Applied Physics Laboratory as part of NASA's Living with a Star (LWS) program (contract NNN06AA01C). Support from the LWS management and technical team has played a critical role in the success of the Parker Solar Probe mission. 


\section{References}

Abbo, L., Ofman, L., Antiochos, S. K., et al. 2016, Space Sci. Rev., 201, 55 Antiochos, S. K., Mikić, Z., Titov, V. S., Lionello, R., \& Linker, J. A. 2011, ApJ, 731,112

Antonucci, E., Romoli, M., Andretta, V., et al. 2020, A\&A, 642, A10 (Solar Orbiter SI)

Arge, C. N., \& Pizzo, V. J. 2000, J. Geophys. Res., 105, 10465

Auchère, F., Andretta, V., Antonucci, E., et al. 2020, A\&A, 642, A6 (Solar Orbiter SI)

Bale, S. D., Goetz, K., Harvey, P. R., et al. 2016, Space Sci. Rev., 204, 49

Bale, S. D., Badman, S. T., Bonnell, J. W., et al. 2019, Nature, 576, 237

Cao, W., Gorceix, N., Coulter, R., et al. 2010, Astron. Nachr., 331, 636

Clette, F., \& Lefèvre, L. 2016, Sol. Phys., 291, 2629

Cranmer, S. R., van Ballegooijen, A. A., \& Edgar, R. J. 2007, ApJS, 171, 520

Culhane, J. L., Harra, L. K., James, A. M., et al. 2007, Sol. Phys., 243, 19

DeForest, C. E., Gibson, S. E., Beasley, M., et al. 2019, AGU Fall Meeting Abstracts, 2019, SH43B-06

De Pontieu, B., Title, A. M., Lemen, J. R., et al. 2014, Sol. Phys., 289, 2733

Fisk, L. A. 1996, J. Geophys. Res., 101, 15547

Fox, N. J., Velli, M. C., Bale, S. D., et al. 2016, Space Sci. Rev., 204, 7

Geiss, J., Gloeckler, G., von Steiger, R., et al. 1995, Science, 268, 1033

Golub, L., Deluca, E., Austin, G., et al. 2007, Sol. Phys., 243, 63

Gómez-Herrero, R., Dresing, N., Klassen, A., et al. 2015, ApJ, 799, 55

Harvey, J. W., Kennedy, J. R., \& Leibacher, J. W. 1987, S\&T, 74, 470

Horbury, T. S., O’Brien, H., Carrasco Blazquez, I., et al. 2020, A\&A, 642, A9 (Solar Orbiter SI)

Howard, R. A., Vourlidas, A., Bothmer, V., et al. 2019, Nature, 576, 232

Howard, R. A., Vourlidas, A., Colaninno, R. C., et al. 2020, A\&A, 642, A13 (Solar Orbiter SI)

Hudson, H., \& Svalgaard, L. 2019, Phys. Today, 72, 10

Kaiser, M. L., Kucera, T. A., Davila, J. M., et al. 2008, Space Sci. Rev., 136, 5

Kasper, J. C., Abiad, R., Austin, G., et al. 2016, Space Sci. Rev., 204, 131

Kasper, J. C., Bale, S. D., Belcher, J. W., et al. 2019, Nature, 576, 228

Kosugi, T., Matsuzaki, K., Sakao, T., et al. 2007, Sol. Phys., 243, 3

Krucker, S., Hurford, G. J., Grimm, O., et al. 2020, A\&A, 642, A15 (Solar Orbiter SI)

Lionello, R., Velli, M., Downs, C., Linker, J. A., \& Mikić, Z. 2014, ApJ, 796, 111

Liu, Z., Xu, J., Gu, B.-Z., et al. 2014, Res. Astron. Astrophys., 14, 705

Maksimovic, M., Bale, S. D., Chust, T., et al. 2020, A\&A, 642, A12 (Solar Orbiter SI)

Martinez Pillet, V., Tritschler, A., Harra, L., et al. 2020, ArXiv e-prints [arXiv:2004.08632]

Matteini, L., Stansby, D., Horbury, T. S., \& Chen, C. H. K. 2019, Nuovo Cimento C Geophysics Space Physics C, 42, 16

McComas, D. J., Angold, N., Elliott, H. A., et al. 2013, ApJ, 779, 2

McComas, D. J., Alexander, N., Angold, N., et al. 2016, Space Sci. Rev., 204, 187

McComas, D. J., Zirnstein, E. J., Bzowski, M., et al. 2017, ApJS, 229, 41

McComas, D. J., Christian, E. R., Cohen, C. M. S., et al. 2019, Nature, 576, 223

Müller, D., St. Cyr, O. C., Zouganelis, I., et al. 2020, A\&A, 642, A1 (Solar Orbiter SI)

Neugebauer, M., \& Liewer, P. C. 2003, J. Geophys. Res. (Space Phys.), 108, 1013

Owen, C. J., Bruno, R., Livi, S., et al. 2020, A\&A, 642, A16 (Solar Orbiter SI)

Panasenco, O., Velli, M., et al. 2013, AIP Conf. Ser., 1539, 50

Panasenco, O., Velli, M., \& Panasenco, A. 2019, ApJ, 873, 25

Panasenco, O., Velli, M., D'Amicis, R., et al. 2020, ApJS, 246, 54

Parker, E.N. 1958, ApJ, 128, 664

Pesnell, W. D., Thompson, B. J., \& Chamberlin, P. C. 2012, Sol. Phys., 275, 3

Rappazzo, A. F., Velli, M., Einaudi, G., \& Dahlburg, R. B. 2005, ApJ, 633, 474

Riley, P., \& Luhmann, J. G. 2012, Sol. Phys., 277, 355

Rochus, P., Auchère, F., Berghmans, D., et al. 2020, A\&A, 642, A8 (Solar Orbiter SI)

Rodríguez-Pacheco, J., Wimmer-Schweingruber, R. F., Mason, G. M., et al. 2020, A\&A, 642, A7 (Solar Orbiter SI)

Scharmer, G. B., Bjelksjo, K., Korhonen, T. K., Lindberg, B., \& Petterson, B. 2003, SPIE Conf. Ser., 4853, 341

Schmidt, W., von der Lühe, O., Volkmer, R., et al. 2012, Astron. Nachr., 333, 796

Seaton, D. B., Berghmans, D., Nicula, B., et al. 2013, Sol. Phys., 286, 43

Shimojo, M., Bastian, T. S., Hales, A. S., et al. 2017, Sol. Phys., 292, 87

Solanki, S. K., Barthol, P., Danilovic, S., et al. 2010, ApJ, 723, L127

Solanki, S. K., Riethmüller, T. L., Barthol, P., et al. 2017, ApJS, 229, 2

Solanki, S. K., del Toro Iniesta, J. C., Woch, J., et al. 2020, A\&A, 642, A11 (Solar Orbiter SI)

SPICE Consortium (Anderson, M., et al.) 2020, A\&A, 642, A14 (Solar Orbiter $\mathrm{SI})$
Stone, E. C., Frandsen, A. M., Mewaldt, R. A., et al. 1998, Space Sci. Rev., 86,

Tritschler, A., Rimmele, T. R., Berukoff, S., et al. 2016, Astron. Nachr., 337, 1064

Tsuneta, S., Ichimoto, K., Katsukawa, Y., et al. 2008, Sol. Phys., 249, 167

von Steiger, R., Schwadron, N. A., Fisk, L. A., et al. 2000, J. Geophys. Res., 105, 27217

Vourlidas, A., Howard, R. A., Plunkett, S. P., et al. 2016, Space Sci. Rev., 204, 83

Walsh, A. P., Horbury, T. S., Maksimovic, M., et al. 2020, A\&A, 642, A5 (Solar Orbiter SI)

Wang, Y. M., \& Sheeley, N. R. J. 1992, in Solar Wind Seven Colloquium, eds. E. Marsch, \& R. Schwenn, 125

Wang, Y. M., \& Sheeley, N. R. J. 2003, ApJ, 587, 818

Wedemeyer, S., Bastian, T., Brajša, R., et al. 2016, Space Sci. Rev., 200, 1

White, S. M., Iwai, K., Phillips, N. M., et al. 2017, Sol. Phys., 292, 88

Wibberenz, G., \& Cane, H. V. 2006, ApJ, 650, 1199

Zhukov, A. 2019, AGU Fall Meeting Abstracts, 2019 SH24A-10

Zouganelis, I., De Groof, A., Walsh, A. P., et al. 2020, A\&A, 642, A3 (Solar Orbiter SI)

1 Earth, Planetary ans Space Sciences, UCLA, Los Angeles, USA e-mail: mvelli@ucla.edu

2 PMOD/WRC, Dorfstrasse 33, 7260 Davos Dorf, Switzerland e-mail: louise.harra@pmodwrc.ch

3 ETH-Zürich, HIT building, Hönggerberg, 8093 Zürich, Switzerland

4 Johns Hopkins University Applied Physics Laboratory, Laurel, MD, USA

e-mail: angelos.vourlidas@jhuapl.edu

5 Department of Physics, University of New Hampshire, Morse Hall Rm 350, Durham, NH 03824, USA

6 Advanced Heliophysics, Pasadena, CA 91106, USA

7 Jet Propulsion LAboratory, California Inst. of Technology, Pasadena, CA 91109, USA

8 European Space Agency, ESTEC, PO Box 299, 2200 AG Noordwijk, the Netherlands

9 European Space Agency, ESAC, Camino Bajo del Castillo s/n, Urb. Villafranca del Castillo, 28692 Villanueva de la Cañada, Madrid, Spain

10 NASA Goddard Space Flight Center, Greenbelt, MD, USA

11 Institut d'Astrophysique Spatiale, CNRS, Univ. Paris-Sud, Université Paris-Saclay, Bât. 121, 91405 Orsay, France

12 Royal Observatory of Belgium, Ringlaan -3- Av. Circulaire, 1180 Brussels, Belgium

13 RAL Space, STFC Rutherford Appleton Laboratory, Harwell, Didcot OX11 0QX, UK

14 Imperial College London, London, UK

15 Naval Research Laboratory, Washington, DC 20375, USA

16 University of Applied Sciences Northwestern Switzerland, Windisch, Switzerland

17 Observatoire de Paris-Meudon, Paris, France

18 UCL-Mullard Space Science Laboratory, University College London, Holmbury St. Mary, Dorking, Surrey RH5 6NT, UK

19 Universidad de Alcalá, Space Research Group, 28805 Alcalá de Henares, Spain

20 Department of Physics and Astronomy, University of Florence, Florence, Italy

21 Max-Planck-Institut für Sonnensystemforschung (MPS), Justusvon-Liebig-Weg 3, 37077 Göttingen, Germany

22 Division for Extraterrestrial Physics, Institute for Experimental and Applied Physics (IEAP), Christian Albrechts University at Kiel, Leibnizstr. 11, 24118 Kiel, Germany

23 Space Sciences Laboratory and Physics Department, University of California, Berkeley, CA, USA

24 University of Michigan, 2455 Hayward Street, Ann Arbor, MI, USA

25 Department of Astrophysical Sciences, Princeton University, Princeton, NJ, USA

26 National Solar Observatory, 3665 Discovery Drive, Boulder, CO, USA

27 School of Space Research, Kyung Hee University, Yongin, Gyeonggi-Do 446-701, Republic of Korea 